Pacific Journal of Mathematics

HAHN-BANACH TYPE THEOREMS FOR HYPOLINEAR
FUNCTIONAL ON REORDERED TOPOLOGICAL VECTOR
SPACES

BERND ANGER AND JöRN LEMBCKE 


\title{
HAHN-BANACH TYPE THEOREMS FOR HYPOLINEAR FUNCTIONALS ON PREORDERED TOPOLOGICAL VECTOR SPACES
}

\author{
BERND ANGer AND JöRN LEMBCKE
}

\begin{abstract}
This article deals with existence and extension theorems for continuous positive linear forms, dominated by hypolinear functionals, i.e., sublinear functionals which may attain the value $+\infty$.

It is proved that a hypolinear functional dominates a continuous positive linear form if and only if its largest increasing and hypolinear minorant exists and is lower semicontinuous at the origin. Conditions are given which imply that any increasing hypolinear functional is lower semicontinuous at the origin.
\end{abstract}

Introduction. This is the second article (cf. [1]) dealing with Hahn-Banach type theorems for hypolinear functionals (i.e., sublinear functionals which may attain the value $+\infty$ ). It contains part of the results announced in [2].

Here we are concerned with existence and extension theorems for continuous positive linear forms on a locally convex space $E$, preordered by a (pointed convex) cone $C$.

Throughout this paper, hypolinear functionals are defined on vector spaces rather than cones. By $[1,1.3(3)]$, this restriction means no loss of generality. As any vector space may be considered as a preordered vector space with equality as order relation, this article generalizes in particular the results of $[1, \S 1-\S 3]$.

Any linear form dominated by an increasing hypolinear functional is positive. On the other hand, the upper envelope of any nonempty set of positive linear forms on $E$ is an increasing hypolinear functional.

These two facts suggest to replace a given hypolinear functional $p$ on $E$ by its largest increasing minorant $\widetilde{p}$, and then to apply the results of [1], provided that $\widetilde{p}$ is also a hypolinear functional. By this procedure, we obtain from theorems on arbitrary linear forms corresponding results for positive linear forms.

After a preliminary first chapter dealing with elementary properties of increasing hypolinear functionals we prove in $\S 2$ that a hypolinear functional $p$ dominates a continuous positive linear form if and only if its largest increasing minorant $\widetilde{p}$ is hypolinear and lower semicontinuous at the origin.

In $\S 3$, we study theorems on the extension of linear forms defined 
on a subspace of $E$ to continuous positive linear forms on $E$. In Theorem 3.4 an additional domination condition is imposed. We use this result to give an alternative proof for a dominated extension theorem of $\mathrm{H}$. Bauer.

The hypolinear functional which is 0 at the origin and $+\infty$ elsewhere dominates every linear form. Therefore, we may apply our results on dominated extension in order to obtain new proofs for extension theorems without domination condition, due to Bauer, Namioka, and Hustad.

In Theorem 4.1, we prove that a numerical function on a preordered locally convex space is the upper envelope of a family of continuous positive linear forms if and only if it is a lower semicontinuous increasing hypolinear functional.

Relations between topology and order structure which ensure that any increasing hypolinear functional is lower semicontinuous at the origin are investigated in $\S 5$.

The last chapter deals with a general type of sandwich theorems. In particular, we generalize a theorem of one of the authors on the existence of positive simultaneous inverse images of linear forms and give a new proof for the theorem of Mazur-Orlicz.

1. Preliminaries. Unless otherwise stated, we shall assume throughout this paper that $E$ is a real locally convex topological vector space and that $C$ is a (pointed convex) subcone of $E$, i.e., $x_{1}, x_{2} \in C$ and $\lambda_{1}, \lambda_{2} \geqq 0$ imply $\lambda_{1} x_{1}+\lambda_{2} x_{2} \in C$.

If $E$ is only an algebraic vector space, then it shall be understood that $E$ is endowed with the finest locally convex topology.

Let $\leqq$ denote the preorder relation defined on $E$ by $C$ (i.e., $x \leqq y$ for $x, y \in E$ if and only if $y-x \in C)$.

Notations 1.1 .

(1) $0 \cdot \infty=\infty \cdot 0=0 \cdot(-\infty)=(-\infty) \cdot 0=0$.

(2) $\inf \{x: x \in \varnothing\}=+\infty$.

(3) For $i=1,2$ let $f_{i}$ be a numerical function on a set $X_{i}$.

If $X_{1}=X_{2}$ and $f_{1}(x) \leqq f_{2}(x)$ for all $x \in X_{1}$, we write $f_{1} \leqq f_{2}$ and say that $f_{2}$ dominates $f_{1}$ ( $f_{1}$ is $f_{2}$-dominated).

If $X$ is a subset of $X_{1} \cap X_{2}$, we denote by $f_{i} \mid X$ the restriction of $f_{i}$ to $X$, and say that $f_{2}$ dominates $f_{1}$ on $X$, if $f_{1}\left|X \leqq f_{2}\right| X$.

As in [1, Definition 1.1] we introduce the notion of a hypolinear functional:

Definition 1.2. A numerical function $p: E \rightarrow \overline{\boldsymbol{R}}$ is called a hypolinear functional, if

(1) $p(x)>-\infty(x \in E)$. 
(2) $p$ is subadditive:

$$
p(x+y) \leqq p(x)+p(y) \quad(x, y \in E) .
$$

(3) $p$ is positively homogeneous:

$$
p(\lambda x)=\lambda p(x) \quad(x \in E, \lambda \geqq 0) .
$$

If in addition $p$ is finite, $p$ is called a sublinear functional.

DeFinition 1.3. For any numerical function $f$ on $E$ we define the numerical functions $\hat{f}, \tilde{f}$, and $\bar{f}$ on $E$ by

and

$$
\begin{aligned}
& \hat{f}(x)=\liminf _{y \rightarrow x} f(y), \\
& \tilde{f}(x)=\inf _{y \geqq x} f(y)
\end{aligned}
$$

$$
\bar{f}(x)=f(-x) .
$$

$\tilde{f}$ is called the regularized of $f$ with respect to the cone $C$.

A numerical function $f$ on a subset $F$ of $E$ is said to be increasing (with respect to $C$ ) if and only if $x, y \in F$ and $x \leqq y$ imply $f(x) \leqq f(y)$.

$f$ is called decreasing if $-f$ is increasing.

$f$ is called positive if $f(x) \geqq 0$ for every $x \in C \cap F$.

The following lemma is easily proved.

LEMMA 1.4. (1) If $f$ and $g$ are numerical functions on $E$ such that $f \leqq g$, then

$$
\hat{f} \leqq \hat{g} \quad \text { and } \quad \widetilde{f} \leqq \widetilde{g}
$$

(2) Let $C^{\prime}$ be a cone in $E$ containing $C$ and $f$ a numerical function on $E$.

Then $\tilde{f} \geqq \tilde{f}^{\prime}$, where $\tilde{f}^{\prime}$ denotes the regularized of $f$ with respect to the cone $C^{\prime}$.

If a numerical function on $E$ is positive, increasing, or decreasing with respect to $C^{\prime}$, then it has the same property with respect to $C$.

Lemma 1.5. For any numerical function $f$ on $E$ we have:

(1) $\hat{f}$ is the largest lower semicontinuous minorant of $f$, and $\hat{\hat{f}}=\hat{f} . f$ is lower semicontinuous at a point $x \in E$ if and only if $f(x)=\hat{f}(x) \dot{\tilde{f}}$

(2) ${ }_{\tilde{f}}$ is the largest minorant of $f$ which is increasing, moreover $\tilde{\tilde{f}}=\widetilde{f} . \quad f$ is increasing if and only if $f=\tilde{f}$. 
(3) $\hat{\tilde{f}}$ is increasing and $\hat{\tilde{f}}=\hat{\hat{f}}=\hat{\hat{\hat{f}}}$. $\hat{\hat{f}}$ is the largest lower semicontinuous increasing minorant of $f$. For $x \in E$

$$
\hat{\tilde{f}}(x)=\sup _{U \in \mathfrak{u}(0)}\left(\inf _{y \in x \rightarrow U+C} f(y)\right),
$$

where $\mathfrak{H}(0)$ denotes the system of neighborhoods of 0 .

(4) $f$ is increasing if and only if $\bar{f}$ is decreasing. Furthermore $\overline{\bar{f}}=f$.

Proof. (1) is well known.

(2) and (4) are obvious.

(3) By (1), (2), and Lemma 1.4(1), any increasing and lower semicontinuous minorant of $f$ is dominated by $\hat{\tilde{f}}$. $\hat{\tilde{f}}$ is lower semicontinuous, by (1). Moreover, we have for $x \in E$,

$$
\begin{aligned}
\hat{\tilde{f}}(x) & =\sup _{U \in \mathfrak{u}(0)}\left[\inf _{z \in x+U}\left(\inf _{c \in C} f(z+c)\right)\right] \\
& =\sup _{U \in \mathfrak{u}(0)}\left(\inf _{y \in x+U+C} f(y)\right) .
\end{aligned}
$$

Hence $\hat{\tilde{f}}$ is also increasing, and therefore $\hat{\tilde{f}}$ is the largest lower semicontinuous and increasing minorant of $f$.

This implies

$$
\hat{\tilde{f}}=\hat{\tilde{\tilde{f}}}=\hat{\hat{\hat{f}}}
$$

By 1.4(1), $\hat{f} \leqq f$ implies $\hat{\hat{\hat{f}}} \leqq \hat{\tilde{f}}$, and $\widetilde{f} \leqq f$ implies $\hat{\tilde{f}}=\hat{\hat{\tilde{f}}} \leqq \hat{\tilde{\hat{f}}}$. Hence $\hat{\tilde{f}}=\hat{\tilde{\hat{f}}}$.

REMARK 1.6. We shall see in Example 1.11(3) that in general $\tilde{\hat{f}}$ need not be lower semicontinuous, i.e., $\tilde{\hat{f}}$ and $\hat{\hat{f}}$ need not coincide, even if $f, \hat{f}, \widetilde{f}, \tilde{\hat{f}}$, and $\hat{\tilde{f}}$ are hypolinear functionals.

Lemma 1.7. A hypolinear functional $p$ on $E$ is increasing if and only if

$$
p(x) \leqq 0 \quad \text { for every } \quad x \in-C
$$

Proof. If $p$ is increasing and $x \in-C$, then $p(x) \leqq p(0)=0$.

Conversely, if $p(x) \leqq 0$ for every $x \in-C$, then we have for $y, z \in E$ with $z \geqq y$

$$
p(y)=p(z+(y-z)) \leqq p(z)+p(y-z) \leqq p(z) .
$$

COROLLARY 1.8. If $p$ is an increasing hypolinear functional 
on $E$, then any p-dominated hypolinear functional (linear form) $q$ on a subspace $F$ of $E$ is increasing (positive).

Proof. For $x \in-C \cap F$ we have

$$
q(x) \leqq p(x) \leqq 0 .
$$

Hence, by 1.7, $q$ is increasing. If $q$ is a linear form, this implies the positivity of $q$.

LeMma 1.9. Let $p$ be a hypolinear functional on $E$.

Then $\widetilde{p}: E \rightarrow \overline{\boldsymbol{R}}$ (cf. Definition 1.3) is an increasing hypolinear functional if and only if $\widetilde{p}$ does not attain the value $-\infty$.

$A$ linear form (a hypolinear functional) $f$ on $E$ is p-dominated and positive (increasing) if and only if it is $\tilde{p}$-dominated.

Proof. It is clear from the definition that $\widetilde{p}$ is increasing and subadditive and that $\widetilde{p}(\lambda x)=\lambda \widetilde{p}(x)$ for $x \in E, \lambda>0$. If $\widetilde{p}$ does not attain $-\infty$, then

$$
-\infty<2 \widetilde{p}(0)=\widetilde{p}(0) \leqq p(0)=0,
$$

hence $\widetilde{p}(0)=0$. Therefore, in this case $\widetilde{p}$ is a hypolinear functional. The converse implication is trivial.

If $f$ is $\widetilde{p}$-dominated, then $f$ is positive (increasing), by 1.8. If $f$ is increasing and $p$-dominated, then for $x, y \in E$ with $y \geqq x$

$$
f(x) \leqq f(y) \leqq p(y),
$$

hence

$$
f(x) \leqq \widetilde{p}(x) .
$$

As any positive linear form is increasing, this proves that lemma.

REMARK 1.10. Another way to prove the preceding lemma would be the following:

Let $I=\{1,2\}, P_{1}=P_{2}=E, p_{1}=p$ and define $p_{2}: P_{2} \rightarrow \overline{\boldsymbol{R}}$ to be 0 on $-C$ and $+\infty$ elsewhere. Then $p_{1}, p_{2}$ are hypolinear functionals and $\widetilde{p}$ is equal to the numerical function $p$ defined in [1, Lemma 1.5] thus 1.9 follows from conditions (6) and (5) of [1, Lemma 1.5] and from 1.7.

Let us now give some examples of increasing hypolinear functionals.

ExAMPles 1.11. (1) Any positive linear form on $E$ is an increasing hypolinear functional.

(2) The upper envelope of any nonempty set of continuous 
positive linear forms on $E$ is a lower semicontinuous increasing hypolinear functional.

(3) Let $p$ be the hypolinear functional of [1, 1.2(1)], which is defined to be 0 at the origin and $+\infty$ elsewhere.

Then $\widetilde{p}$ is an increasing hypolinear functional which is 0 on $-C$ and $+\infty$ elsewhere.

Hence $\hat{\tilde{p}}$ is the lower semicontinuous increasing hypolinear functional which is 0 on the closure $\overline{-C}$ of $-C$ and $+\infty$ elsewhere.

In order to prove Remark 1.6, suppose moreover that $E$ is a Hausdorff locally convex space. Then $p$ is lower semicontinuous, hence $p=\hat{p}$ and $\widetilde{p}=\tilde{\hat{p}}$. Therefore, $\tilde{\hat{p}}$ is 0 on $-C$ and $+\infty$ elsewhere, whereas $\hat{\tilde{p}}$ is 0 on $\overline{-C}$ and $+\infty$ elsewhere. Hence $\hat{\tilde{p}}$ and $\tilde{\hat{p}}$ coincide if and only if the cone $C$ is closed.

(4) Let $I$ be an index set and let $E=\boldsymbol{R}^{(I)}$ be the vector space of [1, Example 1.6].

Then the set $C=\left\{x=\left(x_{i}\right)_{i \in I} \in E: x_{i} \geqq 0\right.$ for all $\left.i \in I\right\}$ is a cone in $E$. Obviously, the hypolinear functional $p$ of $[1$, Example 1.6], defined on $E$ by

$$
p(x)=-\sum_{\substack{i, j \in I \\ i \neq j}} \sqrt{x_{i} x_{j}} \text { for } x=\left(x_{i}\right)_{i \in I} \in C
$$

and by

$$
p(x)=+\infty \text { for } x \notin C,
$$

is decreasing. Hence $\bar{p}$ is an increasing hypolinear functional on $E$.

2. Existence of dominated positive linear forms. The following theorem gives necessary and sufficient conditions for the existence of continuous positive linear forms dominated by a given hypolinear functional $p$. Most of these conditions are formulated in terms of $\widetilde{p}$. If $p$ is increasing (i.e., $p=\widetilde{p}$ ), then the theorem is merely a restatement of [1, Theorem 1.8] in combination with 1.8 .

THeOREM 2.1. Let $p$ be a hypolinear functional on $E$. Then the following assertions are equivalent:

(1) There exists a p-dominated continuous positive linear form $f$ on $E$.

(2) There exists a $\widetilde{p}$-dominated continuous linear form on $E$.

(3) There exists a continuous seminorm (sublinear functional) $q$ on $E$ such that $-q$ is dominated by $\widetilde{p}$.

(4) There is a continuous decreasing sublinear functional $q$ on $E$ such that $-q$ is dominated by $p$.

(5) There is a continuous seminorm $s$ on $E$ such that $x \in E$ and $s(x) \leqq 1$ imply 


$$
p(y) \geqq-1 \text { for all } y \geqq x .
$$

(6) There is a continuous sublinear functional $q$ on $E$ such that $x \in E$ and $q(x) \leqq 1$ imply

$$
p(y) \geqq-1 \text { for all } y \geqq x .
$$

(7) $\widetilde{p}$ is bounded below in some neighborhood of 0.

(8) There is a neighborhood $U$ of 0 such that $p$ is bounded below on $U+C$.

(9) $\tilde{p}(0)=0$ and $\widetilde{p}$ is lower semicontinuous at 0 .

Proof. (1) and (2) are equivalent by 1.9.

Each of the conditions (2), (3), (5), (7), and (9) implies that $\tilde{p}$ is a hypolinear functional. So these conditions are equivalent by $[1,1.8]$, applied to $\widetilde{p}$.

$(1) \Rightarrow(4)$ : We choose $q=-f$. Then $q$ is a continuous decreasing sublinear functional on $E$ and $-q=f$ is $p$-dominated.

$(4) \Rightarrow(3)$ : Let $x, y \in E$ with $y \geqq x$. Then

$$
p(y) \geqq-q(y) \geqq-q(x),
$$

hence

$$
\widetilde{p}(x) \geqq-q(x) .
$$

Therefore, $\widetilde{p}$ dominates $-q$.

Obviously, (5) implies (6).

$(6) \Rightarrow(5)$ : The numerical function $s$ defined on $E$ by

$$
s(x)=\sup \{q(x), q(-x)\}
$$

is a continuous seminorm. For $x \in E$ with $s(x) \leqq 1$, we have $q(x) \leqq 1$, and therefore

$$
p(y) \geqq-1 \quad \text { for every } y \geqq x .
$$

(8) is only a restatement of (7).

CoRollary 2.2. Let $p$ be a hypolinear functional and $q a$ continuous decreasing sublinear functional on $E$ such that $-q$ is p-dominated.

Then there exists a positive linear form $f$ on $E$ such that

$$
-q \leqq f \leqq p \text {. }
$$

Proof. By step $(4) \Rightarrow(3)$ in the proof of the preceding theorem, we have $-q \leqq \widetilde{p}$. Therefore, it follows from [1, Corollary 1.9] that there is a continuous linear form $f$ on $E$ such that $-q \leqq f \leqq \widetilde{p}$. By 
Lemma 1.9, $f$ is positive and $p$-dominated.

3. Extension theorems. We start with a slight formal generalization of a functional defined in [1, cf. 2.1 and $2.2(3)]$.

Definition 3.1. Let $p$ be a numerical function on $E$ and let $f$ be a linear form on a subspace $F$ of $E$.

Then the numerical function $p_{f}$ on $E$ is defined by

$$
p_{f}(x)=\inf _{y \in F}(p(x+y)-f(y)) \text {. }
$$

Lemma 3.2. Let $p$ be a hypolinear functional on $E$ and $f$ a linear form on a subspace $F$ of $E$.

Then we have $\widetilde{p_{f}}=(\widetilde{p})_{f}$, and we write $\widetilde{p}_{f}$ instead of both $\widetilde{p_{f}}$ and $(\widetilde{p})_{f}$.

Moreover, $\widetilde{p}_{f}$ is an increasing hypolinear functional if and only if $\widetilde{p}_{f}$ does not attain the value $-\infty$.

Proof. Let $x \in E$. Then

$$
\begin{aligned}
(\widetilde{p})_{f}(x) & =\inf _{y \in F}\left[\inf _{z \geqq x+y} p(z)-f(y)\right] \\
& =\inf _{y \in F}\left[\inf _{z \geqq x}(p(z+y)-f(y))\right] \\
& =\inf _{z \geqq x}\left[\inf _{y \in F}(p(z+y)-f(y))\right]=\widetilde{p}_{f}(x) .
\end{aligned}
$$

The second part follows from $[1,1.5(6)]$ and 1.9 .

EXAMPLE 3.3. Let $p$ be the hypolinear functional of Example 1.11 (3) which is 0 at the origin and $+\infty$ elsewhere.

Then any hypolinear functional and in particular any linear form defined on a subspace $F$ of $E$ is $p$-dominated.

Hence, by Lemma 1.5, any positive linear form on $E$ is $\widetilde{p}$-dominated, and any continuous positive linear form on $E$ is $\hat{\widetilde{p}}$-dominated.

Let $f$ be a linear form on a subspace $F$ of $E$. Then $p_{f}$ is equal to $f$ on $F$ and to $+\infty$ elsewhere. Hence

$$
\widetilde{p}_{f}(x)=\inf _{\substack{y \in F \\ y \geqq x}} f(y) \quad(x \in E) .
$$

Obviously, $\widetilde{p}_{f}(0)>-\infty$ if and only if $f$ is a positive linear form on $F$.

THEOREM 3.4. For any hypolinear functional $p$ on $E$ and any linear form $f$ on a subspace $F$ of $E$ the following assertions are equivalent: 
(1) $f$ can be extended to a continuous positive linear form on $E$ which is dominated by $p$.

(2) $f$ can be extended to a continuous linear form on $E$ dominated by $\tilde{p}$.

(3) There exists a continuous seminorm (swblinear functional) $q$ on $E$ such that $-q$ is dominated by $\widetilde{p}_{f}$.

(4) There exists a continuous decreasing sublinear functional $q$ on $E$ such that $-q$ is dominated by $p_{f}$.

(5) There is a continuous seminorm (subiinear functional) $q$ on $E$ such that $x \in E$ and $q(x) \leqq 1$ imply

$$
p(z+y)-f(y) \geqq-1 \text { for every } y \in F \text { and every } z \geqq x .
$$

(6) The mapping $\widetilde{p}_{f}$ is bounded below in some neighborhood of 0 .

(7) There is a neighborhood $U$ of 0 such that $p_{f}$ is bounded below on $U+C$.

(8) $\widetilde{p}_{f}(0)=0$ and $\widetilde{p}_{f}$ is lower semicontinuous at 0 .

(9) There is a neighborhood $U$ of 0 such that the set $\{p(z-y)+f(y): y \in F, z \in U+C\}$ is bounded below.

Proof. (1) and (2) are equivalent by 1.9.

In each of the Cases (3)-(8), $p_{f}$ is a hypolinear functional by [1, $2.2(1)]$. Therefore, (3), (4), (5), (6), (7), (8) are respectively equivalent to conditions (3), (4), (5 and 6), (7), (8), (9) of 2.1, applied to $p_{f}$, hence (3)-(8) are equivalent by 2.1. (9) is a restatement of (7).

In each of the Cases (2) and (3), $\widetilde{p}$ is a hypolinear functional. So the equivalence of these conditions follows from [1, Theorem 2.4].

The following extension theorem is due to Bauer [3, Hauptsatz 1 and Bemerkung, p. 181f.].

TheOREM 3.5 (Bauer). Let $p \geqq 0$ be a continuous sublinear functional on $E$ and $f$ a linear form on a subspace $F$ of $E$. Let $V=\{x \in E: p(-x) \leqq 1\}$.

Then $f$ can be extended to a p-dominated continuous positive linear form $g$ on $E$ if and only if

$$
f(y) \geqq-1 \text { for every } y \in F \cap(V+C) .
$$

Proof. (1) If $g$ is a $p$-dominated continuous positive linear extension of $f$, then, for every $y \in F \cap(V+C)$, there is an $x \in V$ such that $y \geqq x$. Hence

$$
f(y)=g(y) \geqq g(x)=-g(-x) \geqq-p(-x) \geqq-1 .
$$


(2) Conversely, suppose

$$
f(y) \geqq-1 \text { for every } y \in F \cap(V+C) .
$$

We shall prove Condition 3.4(5) for $q=\bar{p}$.

Let $x \in V, c \in C$, and $y \in F$. Then

$$
\begin{aligned}
p(x+c+y)-f(y) & \geqq p(c+y)-p(-x)-f(y) \\
& \geqq p(c+y)-f(y)-1 .
\end{aligned}
$$

Therefore, it is sufficient to show

$$
p(c-y)+f(y) \geqq 0 \quad(c \in C, y \in F) .
$$

(i) If $p(c-y)>0$, then $x=(y-c) / p(c-y) \in V$. Hence $y / p(c-y) \in F \cap(V+C)$, and therefore

$$
f\left(\frac{y}{p(c-y)}\right) \geqq-1 \text {. }
$$

This implies

$$
p(c-y)+f(y) \geqq 0 .
$$

(ii) If $p(c-y)=0$, then, for every $\lambda \geqq 0$, we have $\lambda(y-c) \in V$, hence $\lambda y \in F \cap(V+C)$, and therefore $f(\lambda y) \geqq-1$. This implies $f(y) \geqq 0$, hence

$$
p(c-y)+f(y) \geqq p(c-y)=0 .
$$

REMARK 3.6. The preceding theorem is no longer true if $p$ is not supposed to be nonnegative on $E$.

If we choose $E$ to be the real line and define the sublinear functional $p$ on $E$ by $p(x)=-x$, then the linear form $f=0$ on the subspace $F=\{0\}$ trivially satisfies the condition required in Bauer's theorem, but there exists no positive $p$-dominated linear extension of $f$.

The following two theorems on the existence of continuous positive linear extensions of linear forms, without any domination conditions imposed, are respectively due to Bauer [3, Hauptsatz 2] and Namioka [10, Theorem 4.4], and to Hustad [6, Theorem 2]. We shall give new proofs for both theorems, using the fact that any linear form is dominated by the hypolinear functional $p$ of Example 3.3.

Theorem 3.7 (Bauer-Namioka). Let $f$ be a linear form on a subspace $F$ of $E$. There exists a continuous positive linear extension 
of $f$ to $E$ if and only if there exists a neighborhood $U$ of 0 such that the set $f(F \cap(U+C))$ is bounded below.

Proof. Let $p$ be the hypolinear functional of Example 3.3 defined by $p(0)=0$ and by $p(x)=+\infty$ for $x \neq 0$. Any linear form on $E$ is $p$-dominated. Therefore, the result follows from Theorem 3.4, (1) and (9).

Theorem 3.8 (Hustad). A linear form $f$ on a subspace $F$ of $E$ has a continuous positive linear extension to $E$ if and only if $f$ is positive with respect to the cone $C^{\prime}=\overline{f^{-1}(0)+C}$.

Proof. The "only if" part being obvious, let us suppose that $f$ is positive with respect to $C^{\prime}$.

Let $p$ denote the hypolinear functional on $E$ which is 0 at the origin and $+\infty$ elsewhere (cf. 3.3). By 3.4, we have to prove that $\widetilde{p}_{f}$ is bounded below in some neighborhood of 0 .

As in Lemma 1.4(2), we denote by $\widetilde{p}_{f}^{\prime}$ the regularized of $p_{f}$ with respect to the cone $C^{\prime}$. Then $\widetilde{p}_{f}^{\prime} \leqq \widetilde{p}_{f}(1.4(2))$. Hence it is sufficient to show that $\widetilde{p}_{f}^{\prime}$ is bounded below in some neighborhood of 0 .

Suppose, this is not true. By 3.3, we have for $x \in E$

$$
\widetilde{p}_{f}^{\prime}(x)=\inf \left\{f(y): y \in F, y-x \in C^{\prime}\right\} \text {. }
$$

Hence $f$ cannot be the 0 -form on $F$. Therefore, there is a $y_{0} \in F$ such that $f\left(y_{0}\right)=1$.

Let $U$ be a symmetric convex neighborhood of 0 . By the assumption, there is an $x_{U} \in U$ such that $\widetilde{p}_{f}^{\prime}\left(x_{U}\right)<-1$. Hence there is a $y_{U} \in F$ such that $f\left(y_{U}\right)<-1$ and $y_{U}-x_{U} \in C^{\prime} . \quad F$ is the direct sum of $f^{-1}(0)$ and $\boldsymbol{R} \cdot y_{0}$. Hence $y_{U}=\lambda_{U} y_{0}+v_{U}$ for some $\lambda_{U} \in \boldsymbol{R}$ and some $v_{U} \in f^{-1}(0)$. Then also $\lambda_{U} y_{0}-x_{U} \in C^{\prime}$ and $f\left(\lambda_{U} y_{0}\right)<-1$, hence $\lambda_{U}<-1$. $x_{U} \in U$ implies $\lambda_{U} y_{0} \in U+C^{\prime}$. Therefore, also

$$
-y_{0}=\frac{1}{\left|\lambda_{U}\right|}\left(\lambda_{U} y_{0}\right) \in U+C^{\prime} .
$$

As this holds for each symmetric convex neighborhood of 0 , we may conclude $-y_{0} \in C^{\prime}$. Hence $f\left(-y_{0}\right) \geqq 0$, which contradicts $f\left(-y_{0}\right)=-1$.

\section{Approximation of increasing hypolinear functionals.}

THEOREM 4.1. A numerical function $p$ on $E$ is the upper envelope of a nonempty set of continuous positive linear forms on $E$ if and 
only if $p$ is a lower semicontinuous increasing hypolinear functional.

Proof. Let $p$ be a lower semicontinuous increasing hypolinear functional. Then, by [1, Corollary 3.6] $p$ is the upper envelope of all $p$-dominated continuous linear forms, each of which is positive, by 1.8 .

The converse implication follows from Example $1.11(2)$.

CoROLlaRY 4.2. Let $p$ be a hypolinear functional on $E$ dominating at least one continuous positive linear form on $E$.

Then $\hat{\widetilde{p}}$ is the upper envelope of the set of all p-dominated continuous positive linear forms on $E$.

Proof. By 1.5(3), $\hat{\tilde{p}}$ is the largest lower semicontinuous increasing minorant of $p$. Hence a continuous positive linear form on $E$ is $p$-dominated if and only if it is $\hat{\tilde{p}}$-dominated. Therefore, the result follows from 4.1 , applied to the hypolinear functional $\hat{\tilde{p}}$.

Proposition 4.3. The upper envelope of the set of all continuous positive linear forms on $E$ is equal to 0 on $\overline{-C}$ and to $+\infty$ elsewhere.

Proof. As the linear form $f \equiv 0$ on $E$ is continuous and positive, we may apply 4.2 to the hypolinear functional $p$ of Example 1.11(3) which is 0 at the origin and $+\infty$ elsewhere (cf. 3.3).

The following corollary is due to Bauer [3, Satz 2] and Namioka [10, Theorem 4.1], it follows immediately from 4.3.

COROLlaRY 4.4. Let $x_{0} \in E$. There exists a continuous positive linear form $f$ on $E$ with $f\left(x_{0}\right)>0$ if and only if $x_{0}$ does not belong to the closure $\overline{-C}$ of $-C$.

5. Lower semicontinuity of increasing hypolinear functionals. Let $p$ be an increasing hypolinear functional on $E$. Then $p=\widetilde{p}$. In view of Theorem 2.1(9) it would be of great interest to find conditions on the locally convex space $E$ and the cone $C$ which imply that any increasing hypolinear functional on $E$ is lower semicontinuous at 0 .

The similar problem for the continuity of every positive linear form on $E$ has been treated in some well known theorems (cf. 11, Chapter 2, 2.17] or [12, Chapter V, 5.5]).

As the lower semicontinuity of any increasing hypolinear func- 
tional on $E$ at 0 would imply the continuity of any positive linear form on $E$, we shall investigate the conditions given in these theorems.

The proof of the following theorem is similar to the proof of the corresponding results in [11] and [12].

THEOREM 5.1. Each of the following two conditions implies that any increasing hypolinear functional $p$ on $E$ is lower semicontinuous at 0 :

(1) C has nonempty interior $\stackrel{\text { C. }}{\text { (2) }}$

(2) $E$ is a metrizable locally convex space of second category with $E=C-C$, and $C$ is a complete subcone of $E$.

Proof. (1) Let $\varepsilon>0$ and choose $x_{0} \in C^{\circ}$. Then there is a $\lambda>0$ such that $p\left(-\lambda x_{0}\right)>-\varepsilon$. As $p$ is increasing, we get

$$
p(x)>-\varepsilon \text { for every } x \in-\lambda x_{0}+C .
$$

Moreover, as $\lambda x_{0} \in \stackrel{\circ}{C}$,

$$
0 \in-\lambda x_{0}+\stackrel{\circ}{C} \subset-\lambda x_{0}+C .
$$

This implies that $-\lambda x_{0}+C$ is a neighborhood of 0 , bence $p$ is lower semicontinuous at 0 .

(2) As $E$ is metrizable, there exists a countable neighborhood basis $\left(U_{n}\right)_{n \in N}$ of 0 consisting of closed circled sets such that

$$
U_{n+1}+U_{n+1} \subset U_{n} \quad(n \in N) .
$$

By Klee [7] (cf. [11, p. 194]), the sets

$$
V_{n}=U_{n} \cap C-U_{n} \cap C \quad(n \in N)
$$

form a neighborhood base of 0 .

Now, assume that $p$ is an increasing hypolinear functional on $E$ which is not lower semicontinuous at 0 . Then for every $n \in N$ there are elements $x_{n}, y_{n} \in U_{n} \cap C$ such that

$$
p\left(x_{n}-y_{n}\right) \leqq-n
$$

and hence

$$
p\left(-y_{n}\right) \leqq-n .
$$

Since $U_{n+1}+U_{n+1} \subset U_{n}$ for all $n \in N$, the sequence $\left(y_{n}\right)_{n \in N}$ is summable. Let $y=\sum_{n \in N} y_{n}$. As $C$ is closed, we have $y \in C$ and $y \geqq y_{n}$ for all $n \in N$. Therefore, we get

$$
p(-y) \leqq p\left(-y_{n}\right) \leqq-n,
$$




$$
p(-y)=-\infty,
$$

which is impossible.

REMARK 5.2. Condition (2) of Theorem 5.1 holds in particular if $E$ is complete and metrizable, and $C$ is a closed cone generating $E$ (i.e., $E=C-C$ ) (cf. [4, $\S 5, n^{\circ} 3$, Theorem 1]).

REMARK 5.3. By analogy with the corresponding theorem on the continuity of positive linear forms (cf. [12, Chapter V, 5.5. iii]) one might expect that the following condition also implies the lower semicontinuity of any increasing hypolinear functional at 0 :

(3) $E$ is a bornological space (i.e. $E$ is a locally convex space such that each circled convex set absorbing every bounded set is a neighborhood of 0 ) and $C$ is a strict b-cone (i.e., the increasingly directed system of all bounded sets in $E$ is generated by the class $\{B \cap C-B \cap C: B$ is a bounded subset of $E\})$ which is sequentially complete.

However, this conjecture turns out to be false, as we shall show in the following example.

ExAmple 5.4. (cf. [1, Example 1.6] and 1.11(4)). Let $I$ be an uncountable index set, $E=\boldsymbol{R}^{(T)}$ the vector space defined in [1, Example 1.6] endowed with the finest locally convex topology, and $C=\left\{\left(x_{i}\right)_{i \in I} \in E: x_{i} \geqq 0(i \in I)\right\}$.

For the hypolinear functional $p$ of [1, Example 1.6] we have shown that $\bar{p}$ is an increasing hypolinear functional (cf. 1.11(4)) and that $p$ and hence $\bar{p}$ does not dominate any (continuous) linear form on $E$ (cf. [1, Example 1.6(1)]. Hence, by 2.1, $\bar{p}$ is an increasing hypolinear functional on $E$ which is not lower semicontinuous at 0 .

Let us now prove that

(i) $E$ is a bornological space.

(ii) $C$ is a strict b-cone in $E$.

(iii) $C$ is sequentially complete.

(i) Every one-element subset of $E$ is bounded. Hence every circled convex set in $E$ absorbing every bounded set is absorbing and therefore, by the definition of the finest locally convex topology, a neighborhood of 0 .

(ii) A subset $B$ of $E$ is bounded if and only if $B$ is a bounded subset of some finite dimensional subspace $F$ of $E$ (cf. [12, Ch. II, Exercise $7(\mathrm{~b})])$. We may assume that $F=\left\{\left(x_{i}\right)_{i \in I}: x_{i}=0\right.$ for $\left.i \notin J\right\}$ for some finite subset $J$ of $I . \quad F \cap C$ is a strict $b$-cone in $F$ (cf. [11, Chapter II, Example 1.17(a)]). Hence there is a bounded set $B^{\prime}$ in $F$ 
and therefore in $E$ such that $B^{\prime} \cap C-B^{\prime} \cap C \supset B$.

(iii) Any Cauchy sequence in $C$ is bounded and hence contained in some finite dimensional subspace $F$ of $E$. As $F$ is necessarily complete and $C$ is closed, any Cauchy sequence in $C \cap F$ converges to some element of $C \cap F \subset C$.

One might expect that each of the Conditions (1) and (2) of 5.1 even implies that an increasing hypolinear functional on $E$ is lower semicontinuous at any point of $E$. However, the following example shows that this conjecture is not true.

EXAmple 5.5. (cf. [1, Example 5.4]). Let $E$ be the vector space $\mathscr{C}([-1,1])$ of all real-valued continuous functions on the interval $[-1,1]$. We endow $E$ with the supremum norm topology and take for $C$ the cone of all functions in $E$ with values in $\boldsymbol{R}_{+}=[0, \infty)$.

Then (cf. 5.2) $E$ and $C$ satisfy both Conditions (1) and (2) of Theorem 5.1.

We define $g \in E$ by $g(x)=\cos (\pi / 2) x(x \in[-1,1])$ and $p: E \rightarrow \overline{\boldsymbol{R}}$ by

$$
p(h)=\left\{\begin{array}{l}
0, \text { if } h \leqq \lambda g \text { for some } \lambda \geqq 0 \\
+\infty, \text { else }
\end{array}\right\} .
$$

Then $p$ is an increasing hypolinear functional on $E$ (cf. [1, 5.4 and $1.3(3)$ ] and 1.7) which is lower semicontinuous at 0 . But $p$ is not lower semicontinuous at the function $h$, defined on $[-1,1]$ by

$$
h(x)=\sqrt{1-x^{2}} .
$$

We shall now apply our previous results to the case where any increasing hypolinear functional on $E$ is lower semicontinuous at 0 .

THEOREM 5.6. Suppose every increasing hypolinear functional on $E$ is lower semicontinuous at 0 (e.g., if $E$ and $C$ have one of the properties (1), (2) of 5.1).

Then for any increasing hypolinear functional $p$ on $E$ there is a p-dominated continuous positive linear form on $E$.

Proof. The result follows immediately from 2.1 (1 and 9), since $p=\widetilde{p}$.

THEOREM 5.7. Suppose that every increasing hypolinear functional on $E$ is lower semicontinuous at $0(e . g .$, if $E$ and $C$ have one of the properties (1), (2) of 5.1).

Then for any hypolinear functional $p$ on $E$ and any linear form $f$ on a subspace $F$ of $E$ the following conditions are equivalent: 
(1) There exists a p-dominated continuous positive linear form on $E$ which extends $f$.

(2) The mapping $\tilde{p}_{f}$ does not attain the value $-\infty$.

(3) For every $x \in E$, the set

$$
\{p(z+y)-f(y): z \geqq x, y \in F\}
$$

is bounded below.

Proof. By 3.2, (2) implies that $\widetilde{p}_{f}$ is an increasing hypolinear functional, which is lower semicontinuous at 0 , by assumption. Hence (1) and (2) are equivalent by 3.4.

(3) is a restatement of (2).

REMARK 5.8. Suppose that under the assumptions of Theorem 5.7, $p$ is an increasing hypolinear functional. Then $p_{f}=\tilde{p}_{f}$. Hence in this case Condition (2) of 5.7 is the Šaškin-Milman condition (cf. [1, 2.3]). Thus, under, these supplementary assumptions on $E, C$, and $p$, Milman's and Šaškin's result (cf. $[9, \S 1$, Lemma 1]) is true. However, it is not true in general (cf. [1, Remark 2.3]).

6. A sandwich theorem and positive simultaneous inverse images of linear forms. The following lemma generalizes both [1, Lemma 1.5] for vector spaces and [8, Lemma 1.2]. The proof is similar to the proofs of these two lemmas.

LEMma 6.1. Let $I$ be an index set. Suppose that, for $i \in I, E_{i}$ is a vector space, $p_{i}$ a hypolinear functional on $E_{i}$ and $h_{i}: E_{i} \rightarrow E$ a linear mapping.

Then the numerical function $p$ defined on $E$ by

$$
\left({ }^{*}\right) \quad p(x)=\inf \left\{\sum_{i \in J} p_{i}\left(x_{i}\right): J \subset I \text { finite }, x_{i} \in E_{i}, \sum_{i \in J} h_{i}\left(x_{i}\right) \geqq x\right\}
$$

has the following properties:

(1) $p$ is increasing.

(2) $p(x+y) \leqq p(x)+p(y)(x, y \in E$ and $\{p(x), p(y)\} \neq\{+\infty,-\infty\})$.

(3) $p(\lambda x)=\lambda p(x)(x \in E, \lambda>0)$.

(4) $p\left(h_{i}\left(x_{i}\right)\right) \leqq p_{i}\left(x_{i}\right)\left(i \in I, x_{i} \in E_{i}\right)$.

(5) If $p(0) \geqq 0$, then

$$
-p_{i}\left(-x_{i}\right) \leqq p\left(h_{i}\left(x_{i}\right)\right) \quad\left(i \in I, x_{i} \in E_{i}\right) .
$$

(6) An increasing hypolinear functional $q$ on $E$ satisfies

$$
q\left(h_{i}\left(x_{i}\right)\right) \leqq p_{i}\left(x_{i}\right) \quad\left(i \in I, x_{i} \in E_{i}\right)
$$

if and only if $q$ is p-dominated. 
(7) $p$ is an increasing hypolinear functional if and only if $p(x)>-\infty$ for all $x \in E$.

(8) If $p(x)<\infty$ for all $x \in E$ and $p(y)>-\infty$ for some $y \in E$, then $p$ is an increasing sublinear functional.

Proof. (1), (2), (3), and (4) follow immediately from the definition of $p$.

(5) We may assume $p\left(h_{i}\left(x_{i}\right)\right)<\infty$ and, by (4), $p\left(-h_{i}\left(x_{i}\right)\right)<\infty$. Then, by (2),

$$
0 \leqq p(0) \leqq p\left(h_{i}\left(x_{i}\right)\right)+p\left(-h_{\imath}\left(x_{i}\right)\right),
$$

and hence, by (4),

$$
-p_{\imath}\left(-x_{i}\right) \leqq-p\left(h_{i}\left(-x_{i}\right)\right)=-p\left(-h_{\imath}\left(x_{i}\right)\right) \leqq p\left(h_{i}\left(x_{i}\right)\right) .
$$

(6) Let $q$ be an increasing bypolinear functional on $E$ satisfying

$$
q\left(h_{2}\left(x_{i}\right)\right) \leqq p_{2}\left(x_{i}\right) \quad\left(i \in I, x_{i} \in E_{2}\right) .
$$

For $x \in E$, any finite subset $J$ of $I$ and $x_{i} \in E_{i}(i \in J)$ such that $\sum_{i \in J} h_{2}\left(x_{\imath}\right) \geqq x$, we have

$$
q(x) \leqq \sum_{i \in J} q\left(h_{i}\left(x_{i}\right)\right) \leqq \sum_{i \in J} p_{i}\left(x_{i}\right)
$$

Hence $q(x) \leqq p(x)$.

The converse follows from (4).

(7) Let $p(x)>-\infty$ for every $x \in E$. Then $p$ is subadditive, by (2). (3) and (4) imply

$$
2 p(0)=p(0) \leqq 0,
$$

therefore $p(0)=0$. Hence, by (3), $p$ is positively homogeneous.

The converse is trivial.

(8) By (7), we only have to prove

$$
p(x)>-\infty \text { for every } x \in E .
$$

However, $p(x)=-\infty$ for some $x \in E$ implies

$$
p(y) \leqq p(x)+p(y-x)=-\infty,
$$

which contradicts $p(y)>-\infty$.

REMARKS 6.2.

(1) If $E=C-C$, then it is sufficient to show in (7) that $p(x)>-\infty$ for all $x \in-C$, and in (8) that $p(x)<\infty$ for all $x \in C$.

(2) Suppose that, for $i \in I, E_{i}$ is a subspace of $E$ and $h_{i}: E_{i} \rightarrow E$ is the canonical injection. 
Then Lemma 6.1 contains $[1,1.5]$ for vector spaces as a special case for the cone $C=\{0\}$ (i.e., the order relation on $E$ coincides with the equality relation).

We now prove a sandwich theorem for positive linear forms which generalizes [1, Theorem 1.12] for vector spaces.

TheOREM 6.3. Let $K$ and $L$ be disjoint index sets and $I=K \cup L$. For any $i \in I$, let $E_{i}$ be a vector space, $h_{i}: E_{\imath} \rightarrow E$ a linear mapping, and $q_{i}$ a hypolinear functional on $E_{i}$.

Then for any linear subspace $F$ of $E$ and any linear functional $f$ on $F$, the following conditions are equivalent:

(1) There exists a continuous positive linear extension $g$ of $f$ to $E$ such that

$$
-q_{i} \leqq g \circ h_{i} \quad \text { for } \quad i \in K
$$

and

$$
g \circ h_{i} \leqq q_{i} \quad \text { for } \quad i \in L
$$

(2) The numerical function $p$, defined on $E$ by

$$
\begin{array}{r}
p(x)=\inf _{\left\{\sum_{i \in J}\right.} q_{\imath}\left(x_{i}\right)+f(y): J \subset I \text { finite, } x_{i} \in E_{i}, \\
\left.y \in F, \sum_{i \in L \cap J} h_{\imath}\left(x_{i}\right)-\sum_{i \in K \cap J} h_{i}\left(x_{\imath}\right)+y \geqq x\right\},
\end{array}
$$

is bounded below in some neighborhood of 0 .

Proof. Let $i_{0} \notin I$ and $I_{0}=I \cup\left\{i_{0}\right\}$. We define $p_{i}=q_{i}$ for $i \in L$, $p_{i}=\bar{q}_{i}$ for $i \in K, E_{i_{0}}=F, p_{\imath_{0}}=f$, and $h_{i_{0}}: E_{i_{0}} \rightarrow E$ by $h_{i_{0}}(x)=x$. Then $p$ is the numerical function defined in $6.1\left(^{*}\right)$ with $I$ replaced by $I_{0}$.

(1) is equivalent to.

There exists a continuous positive linear form $g$ on $E$ such that

$$
g \circ h_{i} \leqq p_{i} \quad\left(i \in I_{0}\right) .
$$

By $6.1(7)$ and $2.1,(2)$ is equivalent to

(4) There is a p-dominated continuous positive linear form $g$ on $E$.

By 6.1(6), (3) and (4) are equivalent.

Corollary 6.4. Let $K$ and $L$ be disjoint index sets and $I=K \cup L$. For $i \in I$ let $E_{i}$ be a vector space, $h_{i}: E_{i} \rightarrow E$ a linear mapping, and $q_{i}$ a hypolinear functional on $E_{i}$.

Suppose, there exists a $j \in I$ with $E_{j}=E$ such that $h_{j}$ is the 
identity and $q_{j}$ is a continuous sublinear functional on $E$.

Then, for any linear subspace $F$ of $E$ and any linear form $f$ on $F$, the following conditions are equivalent:

(1) There exists a continuous positive linear extension $g$ of $f$ to $E$ such that

$$
-q_{i} \leqq g \circ h_{i} \quad \text { for } \quad i \in K
$$

and

$$
g \circ h_{i} \leqq q_{i} \text { for } i \in L .
$$
that

(2) For any finite subset $J$ of $I, x_{i} \in E_{i}(i \in J)$, and $y \in F$ such

$$
\sum_{i \in L \cap J} h_{\imath}\left(x_{i}\right)-\sum_{i \in K \cap J} h_{i}\left(x_{i}\right) \geqq y,
$$

we have

$$
\sum_{i \in J} q_{i}\left(x_{i}\right) \geqq f(y)
$$

Proof. (1) is identical to Condition 6.3 (1).

If $p$ denotes the numerical function defined in 6.3 (2), then (2) is equivalent to

$$
p(0) \geqq 0 .
$$

By $6.1(3), 6.3$ (2) implies (3).

Conversely, if (3) holds, then by 6.1(5),

$$
-q_{j} \leqq p \circ h_{j}=p \quad \text { if } \quad j \in K
$$

and

$$
-\bar{q}_{j} \leqq p \circ h_{j}=p \quad \text { if } \quad j \in L .
$$

By the assumption on $q_{j}$, this implies $6.3(2)$.

CoRollary 6.5 (Theorem of Mazur-Orlicz). Let $p$ be a continuous sublinear functional on $E$ and $K$ an index set. Let $\left(x_{k}\right)_{k \in K}$ and $\left(\rho_{k}\right)_{k \in K}$ be families of elements of $\boldsymbol{E}$ and $\boldsymbol{R}$, respectively.

Then there is a p-dominated continuous positive linear form $g$ on $E$ such that

$$
\rho_{k} \leqq g\left(x_{k}\right) \quad(k \in K)
$$

if and only if for any finite subset $J$ of $K$, any family $\left(\lambda_{k}\right)_{k \in J}$ of nonnegative real numbers and any $x \in E$ with $\sum_{k \in J} \lambda_{k} x_{k} \leqq x$, we have

$$
\sum_{k \in J} \lambda_{k} \rho_{k} \leqq p(x)
$$


Proof. We choose $j \notin K, L=\{j\}, I=K \cup L, E_{j}=E, q_{j}=p$, $E_{k}=\boldsymbol{R} \cdot x_{k}(h \in K)$ and define $q_{k}: \bar{E}_{k} \rightarrow \boldsymbol{R}$ by

$$
q_{k}\left(\lambda x_{k}\right)=\left\{\begin{aligned}
-\lambda \rho_{k}, & \lambda \geqq 0 \\
\infty, & \lambda<0
\end{aligned}\right\} .
$$

Moreover, for every $i \in I$, let $h_{i}: E_{i} \rightarrow E$ be the canonical injection.

Then, for every $i \in I, q_{i}$ is a hypolinear functional on $E_{i}$, and $q_{j}$ is a continuous sublinear functional.

We may therefore apply Corollary 6.4 to the linear form $f=0$ on the subspace $F=\{0\}$ of $E$, and get the equivalence of the following two conditions:

(1) There is a continuous positive linear form $g$ on $E$ (which extends $f$ ) such that

$$
-q_{k}\left(\lambda x_{k}\right) \leqq g\left(\lambda x_{k}\right) \quad(k \in K, \lambda \in R)
$$

and

$$
g(x) \leqq p(x) \quad(x \in E)
$$

(2) For any finite subset $J$ of $K$, any family $\left(\lambda_{k}\right)_{k \in J}$ of real numbers, and any $x \in E$ such that $x-\sum_{k \in J} \lambda_{k} x_{k} \geqq 0$,

$$
p(x)+\sum_{k \in J} q_{k}\left(\lambda_{k} x_{k}\right) \geqq 0 .
$$

Obviously, (1) and (2) are equivalent to the first and second condition of Corollary 6.5, respectively.

In $[8,1.3]$ the usual Hahn-Banach theorem has been used to prove an existence theorem for positive simultaneous inverse images of linear forms. This theorem was used in [8] to solve some questions in measure theory. As a third corollary of Theorem 6.3 we show how the artificial domination Condition (C) of $[8, \S 1]$ can be dropped by introducing a boundedness Condition $6.6(2)$ which is weaker then the combination of (C) and $[8,1.3(3)$ or (4)].

Corollary 6.6. Let $E$ be a locally convex space and $C$ a subcone of $E$. Let $I$ be an index set. Suppose, for every $i \in I$, there is

(i) a vector space $E_{i}$,

(ii) a linear mapping $h_{i}: E_{i} \rightarrow E$,

(iii) a linear form $f_{i}$ on $E_{i}$.

We define the numerical function $p$ on $E$ by

$$
p(x)=\inf \left\{\sum_{i \in J} f_{i}\left(x_{i}\right): J \subset I \text { finite, } x_{i} \in E_{i}, \sum_{i \in J} h_{i}\left(x_{i}\right) \geqq x\right\} .
$$

Then the following assertions are equivalent: 

that

(1) There is a continuous positive linear form $g$ on $E$ such

$$
f_{i}=g \circ h_{i} \quad(i \in I) .
$$

(2) $p$ is bounded below in some neighborhood of 0 .

Proof. In Theorem 6.3 we choose $L=I, K=\varnothing, q_{i}=f_{i}, F=\{0\}$ and $f=0$. Then $p$ is the numerical function defined in $6.3(2)$.

(2) is identical to $6.3(2)$.

Obviously, (1) implies 6.3 (1).

Conversely, 6.3 (1) implies for $i \in I$ and $x_{i} \in E_{i}$,

$$
f_{i}\left(x_{\imath}\right)=-f_{\imath}\left(-x_{\imath}\right) \leqq-g\left(h_{\imath}\left(-x_{i}\right)\right)=g\left(h_{i}\left(x_{\imath}\right)\right) \leqq f_{\imath}\left(x_{i}\right) \text {, }
$$

and therefore (1).

\section{REFERENCES}

1. B. Anger and J. Lembcke, Hahn-Banach type theorems for hypolinear functionals, Math. Ann., 209 (1974), 127-151.

2. - Une généralisation du théorème de Hahn-Banach aux fonctions souslinéaires à valeurs numériques, C. R. Acad. Sci. Paris, Sér. A 277 (1973), 509-511.

3. H. Bauer, Über die Fortsetzung positiver Linearformen, Bayer. Akad. Wiss. Math.-Natur. Kl. S.-B., (1957), 177-190.

4. N. Bourbaki, Topologie générale, Chap. IX, Hermann, Paris, 1948.

5. G. Choquet, Lectures on Analysis, vol. I, Benjamin, New York-Amsterdam, 1969.

6. O. Hustad, Linear inequalities and positive extension of linear functionals, Math. Scand., 8 (1960), 333-338.

7. V. L. Klee Jr., Boundedness and continuity of linear functionals, Duke Math. J., 22 (1955), 263-269.

8. J. Lembcke, Gemeinsame Urbilder endlich additiver Inhalte, Math. Ann., 198 (1972), 239-258.

9. D. P. Milman, Facial characterization of convex sets, Trans. Moskow Math. Soc., 22 (1970), 69-139.

10. J. Namioka, Partially ordered linear topological spaces, Amer. Math. Soc. Memoir, No. 24, Providence, 1957.

11. A. L. Peressini, Ordered Topological Vector Spaces, Harper \& Row, New York, 1967.

12. H. Schaefer, Topological Vector Spaces, Macmillan, New York, 1966.

Received April 26, 1973. This research was done while the authors were postdoctoral fellows of the NRC of Canada at the University of British Columbia. 



\section{PACIFIC JOURNAL OF MATHEMATICS}

\section{EDITORS}

RICHARD ARENS (Managing Editor)

University of California

Los Angeles, California 90024

R. A. Beaumont

University of Washington

Seattle, Washington 98105
J. DugundJI

Department of Mathematics University of Southern California Los Angeles, California 90007

D. Gilbarg AND J. Milgram Stanford University

Stanford, California 94305

\section{ASSOCIATE EDITORS}
E. F. BECKENBACH
B. H. NeUmanN
F. WOLF
K. YOSHIDA

\section{SUPPORTING INSTITUTIONS}

UNIVERSITY OF BRITISH COLUMBIA CALIFORNIA INSTITUTE OF TECHNOLOGY

UNIVERSITY OF CALIFORNIA

MONTANA STATE UNIVERSITY

UNIVERSITY OF NEVADA

NEW MEXICO STATE UNIVERSITY

OREGON STATE UNIVERSITY

UNIVERSITY OF OREGON

OSAKA UNIVERSITY
UNIVERSITY OF SOUTHERN CALIFOF

STANFORD UNIVERSITY

UNIVERSITY OF TOKYO

UNIVERSITY OF UTAH

WASHINGTON STATE UNIVERSITY UNIVERSITY OF WASHINGTON

AMERICAN MATHEMATICAL SOCIET NAVAL WEAPONS CENTER 


\section{Pacific Journal of Mathematics}

\section{Vol. 54, No. 1 \\ May, 1974}

Ralph K Amayo, Engel Lie rings with chain conditions ..................

Bernd Anger and Jörn Lembcke, Hahn-Banach type theorems for hypolinear

functionals on preordered topological vector spaces ..................

Gregory Frank Bachelis and Samuel Ebenstein, On $\Lambda(p)$ sets ................

Harvey Isaac Blau, Indecomposable modules for direct products of finite

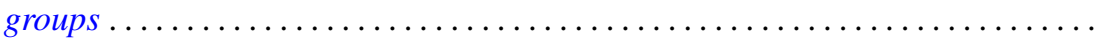

Larry Eugene Bobisud and James Calvert, Singular perturbation of a

time-dependent Cauchy problem in a Hilbert space ................

Walter D. Burgess and Robert Raphael, Abian's order relation and orthogonal

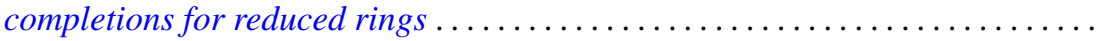

James Diederich, Representation of superharmonic functions mean continuous at

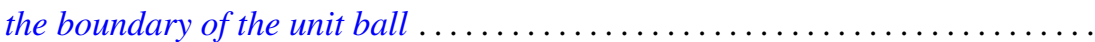

Aad Dijksma and Hendrik S. V. de Snoo, Self-adjoint extensions of symmetric

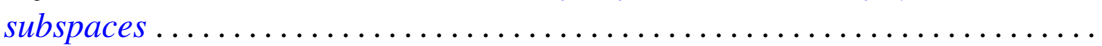

Gustave Adam Efroymson, A Nullstellensatz for Nash rings . . . . . . . . . . . . .

John D. Elwin and Donald R. Short, Branched immersions onto compact orientable surfaces . . . . . . . . . . . . . . . . . . . . . . . . .

John Douglas Faires, Comparison of the states of closed linear

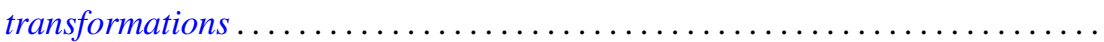

Joe Wayne Fisher and Robert L. Snider, On the von Neumann regularity of rings with regular prime factor rings .

Franklin Takashi Iha, A unified approach to boundary value problems on compact intervals

Palaniappan L. Kannappan and Che Tat $\mathrm{Ng}$, On functional equations connected with directed divergence, inaccuracy and generalized directed divergence

Samir A. Khabbaz and Elias Hanna Toubassi, The module structure of Ext $(F, T)$ over the endomorphism ring of $T$...

Garo K. Kiremidjian, On deformations of complex compact manifolds with boundary.

Dimitri Koutroufiotis, Mappings by parallel normals preserving principal

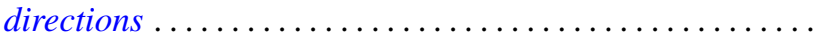

W. K. Nicholson, Semiperfect rings with abelian adjoint group

Norman R. Reilly, Extension of congruences and homomorphisms to translational hulls

Sadahiro Saeki, Symmetric maximal ideals in $M(G)$

Brian Kirkwood Schmidt, On the homotopy invariance of certain functors ...

H. J. Shyr and T. M. Viswanathan, On the radicals of lattice-ordered rings ...

Indranand Sinha, Certain representations of infinite group algebras ...

David Smallen, The group of self-equivalences of certain complexes ...

Kalathoor Varadarajan, On a certain problem of realization in homotopy

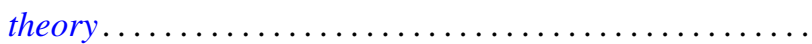

